\title{
Micromanaging osteoporosis
}

New research has revealed that the microRNA miR-34a is a key inhibitor of osteoclastogenesis, with therapeutic potential in the treatment of osteoporosis and to prevent bone metastases. "miR-34a was implicated as a tumour suppressor in primary tumours in earlier studies; now we have discovered a new physiological role for miR-34a in the skeletal system," notes author Yihong Wan from The University of Texas Southwestern Medical Center, USA.

Altered levels of osteoclastogenesis contribute to the development of osteoporosis. Moreover, previous work has shown that osteoclasts can also promote bone metastasis (a complication of many different types of cancer). Using a combination of pharmacological, genetic and disease mouse models,
Wan and colleagues investigated the role of miR-34a during osteoclastogenesis.

The investigators found that miR-34a expression was downregulated during osteoclast differentiation. Crucially, in transgenic mice that overexpressed miR-34a in osteoclasts, bone resorption was reduced and bone mass (as measured

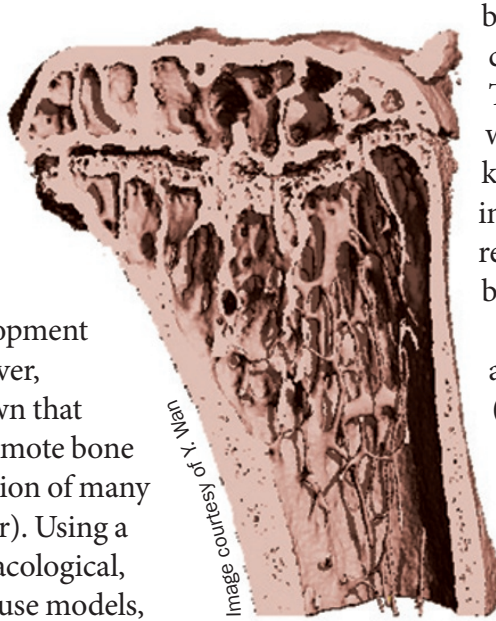
by micro-CT) was increased compared with controls. The opposite scenario was observed in miR-34aknockout mice, which had increased levels of bone resorption and reduced bone mass.

Mechanistically, the study authors pinpointed Tgif2 (transforming growth factor$\beta$-induced factor 2) as a key target of miR-34a that is pro-osteoclastogenic; Tgif2-knockout mice had reduced bone resorption and increased bone mass versus controls, and deletion of Tgif2 in vitro in osteoclast cultures abolished miR-34a regulation.

Interestingly, ovariectomy-induced osteoporosis and bone metastasis (from breast and skin tumours) are diminished in transgenic mice overexpressing miR-34a in osteoclasts. Furthermore, treatment with nanoparticles containing miR-34a attenuated ovariectomy-induced osteoporosis and bone metastasis in mice.

"miRNAs may be a novel class of RNAbased therapies for bone disease," says Wan. The role of microRNAs in bone physiology is just emerging, and the researchers plan further work to explore the therapeutic potential of their discovery.

\section{Katrina Ray}

Original article Krzeszinki, J. Y. et al. miR-34a blocks osteoporosis and bone metastasis by inhibiting osteoclastogenesis and Tgif2. Nature doi:10.1038/ nature 13375 